\title{
Editorial for the special section on implicit surfaces
}

\author{
Brian Wyvill \\ University of Calgary, Dept. of Computer Science, 2500 University Drive N.W., Calgary, Alberta, Canada T2N 1N4
}

This special section is the first of two that will be devoted to a topic of growing importance, that is the field of implicit surfaces or implicit modelling as it is also known. The first of these papers, describes a fast adaptive algorithm for polygonizing an implicit surface. The central idea of the Shrinkwrap algorithm can be explained conveniently as an example of homotopy. Indeed, the purpose is to stabilize a numerical algorithm for finding points on an implicit surface, defined by a function $f$. Instead of doing this in one step, the shrinkwrap algorithm takes a series of functions $f_{i}$ that smoothly interpolate between a trivial first function $f_{0}$ and the final function $f$. The functions $f_{i}$ constitute the homotopic relation between $f_{0}$ and $f$.

The demands on modelling systems increase as there is an expectation from users to have more expressive power to build more complex models. Therefore it is important that modeling techniques can cope with the complexity of both CSG and implicit blending. In this light, the second paper makes use of advantages offered by implicit surfaces of automatic blending and introduces a new way of combining blending and CSG operations.

In the third paper, the authors put a new light on an old problem. Much research has gone into the problem of reconstructing a three-dimensional surface from data-sets. The method described, creates an implicit-surface from the input data.

Professor Brian Wyvill, University of Calgary Special section editor 